\title{
EXISTENCE AND STABILITY THEOREMS FOR ABSTRACT PARABOLIC EQUATIONS, AND SOME OF THEIR APPLICATIONS
}

\author{
GERHARD STRÖHMER \\ Department of Mathematics, University of Iowa \\ Iowa City, Iowa 52242, U.S.A. \\ E-mail: strohmer@math.uiowa.edu \\ WOJCIECH ZAJA̧CZKOWSKI \\ Institute of Mathematics, Polish Academy of Sciences \\ P.O. Box 13\%, 00-950 Warszawa, Poland \\ E-mail: wmzajacz@impan.gov.pl
}

\begin{abstract}
For a class of semi-abstract evolution equations for sections on vector bundles on a three-dimensional compact manifold we prove that for initial values with certain symmetries strong solutions exist for all times. In case these solutions become small after some time, strong solutions exist also for small perturbations of these initial values. Many systems from fluid mechanics are included in this class.
\end{abstract}

1. Introduction. While, given large sufficiently smooth initial values, the timedependent Navier-Stokes equations have classical solutions in two space dimensions (see $[9],[4])$, it is not known whether this is true in general in three space dimensions. Only for flows having certain symmetries classical solutions are known to exist (see [5], [10]). These two papers prove existence of classical solutions for two quite different symmetries, and with quite different methods.

As in the arguments given in [2] and [16] for two-dimensional flows only the validity of certain imbedding theorems seems to play a role, it appears worthwhile to try to prove such an existence theorem for a general class of problems and symmetries. This is the first aim of this paper.

In [11] it was proved that if $\mathbf{v}(x, t)$ is the velocity field of a strong solution of the Navier-Stokes equation, and

1991 Mathematics Subject Classification: 35B35, 35K50, 47D03.

The paper is in final form and no version of it will be published elsewhere. 


$$
\int_{0}^{\infty}\|\nabla \mathbf{v}(t)\|_{L_{4}}^{4} d t<\infty
$$

then for all initial states that are sufficiently close to $\mathbf{v}(x, 0)$ exactly one classical solution of the Navier-Stokes equation exists. The second aim of the paper is to prove similar, though not identical, theorems in a more abstract form suitable for many applications. These results can be applied in the cases quoted above and at the same time are applicable to the Boussinesq approximation of compressible flow and incompressible magneto-hydrodynamics. Examples of this are discussed in Sec. 4. In the main part we consider the partly abstract parabolic equation

$$
U_{t}+A U=F(U, t)
$$

where $A$ is a self-adjoint positive operator with domain $D(A)$ on a Hilbert space $H$. The space $H$ is a closed subspace of the space $L_{2}(\mathcal{V})$ of square integrable sections of a vector bundle $\mathcal{V}$ based on a compact $C^{\infty}$ manifold $\mathcal{M}$ of dimension three with a $C^{3}$-boundary. As $\mathcal{M}$ is compact this space can be defined in a natural manner (see Sec. 1.1). We denote its scalar product by $(\cdot, \cdot)$ and its norm by $\|\cdot\|$. The language of vector bundles (see chap. 4, Sec. 1 (pp. 85-92) in [3]) is only used here in order to be able to cover the case of partly periodic boundary conditions. (E.g. periodic flow in an infinite pipe.) So in a first reading one may want only to think of spaces of functions from a bounded $C^{3}$-domain $\Omega$ in $\mathbb{R}^{3}$ to $\mathbb{R}^{n}$. We can of course think of $\mathcal{V}$ as the union $\bigcup_{m \in \mathcal{M}} \mathcal{V}_{m}$, where $\mathcal{V}_{m}$ is the fiber of $\mathcal{V}$ at $m \in \mathcal{M}$.

We also have a natural definition of the spaces $L_{p}$, and $H_{p}^{i}(\mathcal{V})$ for $i=1,2$, and $p \in[1, \infty)$. We assume that $D(A)$ is a closed subset of $H_{2}^{2}(\mathcal{V}) \cap H$, and that $\|A U\|$ is an equivalent norm to $\|\cdot\|_{H_{2}^{2}}$ on $D(A)$, and $\left\|A^{1 / 2} U\right\|$ is also equivalent to $\|U\|_{H_{2}^{1}}$ on $D\left(A^{1 / 2}\right)$. Then $A$ has a discrete spectrum as $\mathcal{M}$ is compact. Let $S=\left\{T_{\omega}: \omega \in \mathbb{R}\right\}$ with $T_{0}=i d_{\mathcal{V}}$ be a compact one-dimensional group of vector-bundle isomorphisms of $\mathcal{V}$ which depend infinitely differentiably on $\omega$. There is also a group of transformations $\widetilde{T}_{\omega}$ from $\mathcal{M}$ to itself such that $T_{\omega}$ covers $\widetilde{T}_{\omega}$. We assume $d \widetilde{T}_{\omega}(m) / d \omega \neq 0$ everywhere in $\mathcal{M}$. Now let $L_{\omega}$ be defined by

$$
L_{\omega} U(x)=T_{\omega} U\left(\widetilde{T}_{\omega}^{-1}(x)\right) .
$$

Then $\widetilde{H}=\left\{U \in H: L_{\omega} U=U\right.$ for all $\left.\omega \in \mathbb{R}\right\}$ is easily seen to be a closed subspace of $L_{2}(\mathcal{V})$. Let us assume $L_{\omega}(D(A))=D(A), L_{\omega} A=A L_{\omega}$, and that $F: H_{5}^{1}(\mathcal{V}) \times[0, \infty) \rightarrow H$ has the property $F\left(L_{\omega} U, t\right)=L_{\omega} F(U, t)$. Also let $\lambda_{0}=\inf _{\|U\|=1}(A U, U)$, and for $a \in \mathbb{R}$ let $a^{+}=\max (0, a)$, and $a^{-}=\max (0,-a)$.

Assume we are given numbers $\eta \in[0,1), C_{3}, C_{6}<\infty, \nu_{1}, \nu_{2} \in[0,1)$ with $\nu_{1}+\nu_{2}<\frac{3}{2}$ such that

$$
(F(U, t), U)^{+} \leq \eta\left[(F(U, t), U)^{-}+\left\|A^{1 / 2} U\right\|^{2}\right]+C_{1}(t)\|U\|^{2}+C_{2}(t)
$$

with some functions $C_{1}(t), C_{2}(t) \leq C_{6}$,

$$
\|F(U, t)\| \leq C_{3}\left(\|U\|_{L_{4}}+1\right)\left(\|U\|_{H_{4}^{1}}+1\right)
$$

and 


$$
\left\|F(U, t)-F\left(U^{\prime}, t^{\prime}\right)\right\| \leq C_{5}\left(\|U\|_{H_{5}^{1}}+\left\|U^{\prime}\right\|_{H_{5}^{1}}\right)\left(\left\|U-U^{\prime}\right\|_{H_{5}^{1}}+\left|t-t^{\prime}\right|\right)
$$

with an increasing function $C_{5}$.

We call a function $U:\left[t_{0}, t_{1}\right] \rightarrow H$ a solution of $(E Q)$ on the interval $\left[t_{0}, t_{1}\right]$ if $U$ belongs to $C^{0}\left(\left[t_{0}, t_{1}\right], H_{5}^{1}\right) \cap C^{0}\left(\left(t_{0}, t_{1}\right], D(A)\right) \cap C^{1}\left(\left(t_{0}, t_{1}\right], L_{2}\right)$ and solves $(E Q)$ on $\left(t_{0}, t_{1}\right]$.

We obtain the following results.

THEOREM 1. For every $U_{0} \in \widetilde{H} \cap D(A)$ there is a solution $U(t) \in \widetilde{H}$ of $(E Q)$ for all times.

THEOREM 2. There is a number $\delta>0$ such that if there is a $K_{1}<\infty$ with $\max \left(C_{1}(t), C_{2}(t)\right) \leq \delta$ for $t \geq K_{1}$, then for every solution $U(t)$ of $(E Q)$ on $[0, \infty)$ there is an $\varepsilon>0$ such that if $W_{0} \in D(A)$ and $\left\|W_{0}-U(0)\right\|_{H_{2}^{2}}<\varepsilon$, then the solution $W(t)$ of $(E Q)$ with initial value $W_{0}$ also exists for all times.

The proofs of these theorems can be split up into two clearly distinct parts. First we prove two somewhat more general theorems dealing with this situation in a more abstract setting, and then we show that the abstract theory is applicable to the semi-abstract situation described above. The more abstract section may have other applications also. In Sec. 2 we use some ideas from [2] (see also [16], III.3.1), formulated in the style of and using some techniques from [18] and [15]. In Sec. 3 we then show how our symmetry group allows us to eliminate one independent variable, and thus permits us to use twodimensional imbedding theorems for all symmetric functions. Sec. 4 then chiefly discusses the application of these abstract theorems to the equations of magneto-hydrodynamics.

1.1. Notation and function spaces. The letter $C$ is used for generic positive constants with varying values. The norm of any Banach space $B$ is denoted by $\|\cdot\|_{B}$. In the notation for the function spaces defined below we will often leave out the designation of domain and range, if no confusion can arise from this. Let $S \subset \mathbb{R}^{m}$ be a bounded open set or its closure. Then $C^{k+\alpha}\left(S, \mathbb{R}^{n}\right)(k=0,1,2, \ldots, \alpha \in(0,1))$ are the spaces of all real vector-valued functions that are, locally in $S$, Hölder-continuous with exponent $\alpha$, the same being true for the derivatives up to order $k$. This means that these are Banach spaces with the usual norms only in the case of $S$ compact.

Then if $\Omega$ is open, $L^{p}(\Omega)$ is the closure of $C^{0}(\bar{\Omega})$ with respect to the norm $\left(\int_{\Omega}|u(x)|^{p} d x\right)^{1 / p} . L^{2}$ is of course a Hilbert space, and regardless of domain and range we will denote its scalar product by $(\cdot, \cdot)$. For any positive integer $k$ we denote by $H_{p}^{k}(\Omega)$ the set of all functions that have distributional derivatives in $L^{p}(\Omega)$ up to order $k$. For other $\nu \geq 0$ we define these spaces by complex interpolation. We will often write $H_{2}^{\nu}$ as $H^{\nu}$.

Now we must devote some attention to the definition of function spaces for sections on $\mathcal{V}$. As $\mathcal{M}$ is a compact manifold, we can find a finite collection of relatively open subsets $O_{i}$ of $\mathbb{R}^{n+}=\left\{x \in \mathbb{R}^{n}: x_{n} \geq 0\right\}$ and $C^{3}$-embeddings $\mathcal{T}_{i}: \bar{O}_{i} \rightarrow \mathcal{M}(i=1, \ldots, m)$ such that $\bigcup_{i=1}^{m} \mathcal{T}_{i}\left(O_{i}\right)=\mathcal{M}$. For any section $V$ the composition $V\left(\mathcal{T}_{i}(x)\right): O_{i} \rightarrow \mathcal{V}$ can be considered as a vector function on $O_{i}$, and we can then define the norm for sections as

$$
\|V\|_{B}=\sum_{i=1}^{m}\left\|V \circ \mathcal{T}_{i}\right\|_{B}
$$

for any function space $B$. 
2. Existence and stability theorems for abstract evolution equations. In this chapter we consider a somewhat more abstract situation, but we borrow heavily from the notation so far introduced. Objects with the same notation will play entirely analogous roles, although the constants denoted by the same letters will not necessarily have the same values. Again we are considering an equation

$$
U_{t}+A U=F(U, t)
$$

where now $A$ is a positive self-adjoint operator on an abstract Hilbert space $H$ with a domain of definition $D(A)$, and $F: D(A) \times[0, \infty) \rightarrow H$. We assume that $A$ has a compact inverse and we define also

$$
\lambda_{0}=\inf _{\|U\|=1}(A U, U) .
$$

Assume we are given numbers $\eta \in[0,1), \mu \in\left[\frac{1}{2}, 1\right), C_{6}<\infty, \nu_{1}, \nu_{2} \in[0,1)$ with $\nu_{1}+\nu_{2}<\frac{3}{2}$, such that

$$
(F(U, t), U)^{+} \leq \eta\left[(F(U, t), U)^{-}+\left\|A^{1 / 2} U\right\|^{2}\right]+C_{1}(t)\|U\|^{2}+C_{2}(t)
$$

with some functions $C_{1}(t), C_{2}(t) \leq C_{6}$,

$$
\|F(U, t)\| \leq C_{3}\left(\left\|A^{1 / 2} U\right\|\right)\left(\left\|A^{\mu} U\right\|+1\right),
$$

and

$$
\left\|F(U, t)-F\left(U^{\prime}, t^{\prime}\right)\right\| \leq C_{5}\left(\left\|A^{\mu} U\right\|+\left\|A^{\mu} U^{\prime}\right\|\right)\left(\left\|A^{\mu}\left(U-U^{\prime}\right)\right\|+\left|t-t^{\prime}\right|\right)
$$

with increasing functions $C_{3}, C_{5}$. We can of course immediately extend $F$ to $[0, \infty) \times$ $D\left(A^{\mu}\right)$ by virtue of inequality (6), and from now on we will denote this extension by $F$. We call a function $U:\left[t_{0}, t_{1}\right] \rightarrow H$ a solution of $(E Q)$ if $U$ belongs to $C^{0}\left(\left[t_{0}, t_{1}\right], D\left(A^{\mu}\right)\right) \cap$ $C^{0}\left(\left(t_{0}, t_{1}\right], D(A)\right) \cap C^{1}\left(\left(t_{0}, t_{1}\right], H\right)$, and solves $(E Q)$ on $\left(t_{0}, t_{1}\right]$. Let $S=\left\{L_{\omega}: \omega \in \mathbb{R}\right\}$ also be a group of linear isomorphisms of $H$ into itself, and also $L_{\omega} D(A)=D(A), A L_{\omega}=L_{\omega} A$, and $L_{\omega} F(U, t)=F\left(L_{\omega} U, t\right)$ for all $\omega \in \mathbb{R}$. Let also $\widetilde{H}=\left\{U \in H: L_{\omega} U=U\right.$ for all $\omega \in \mathbb{R}\}$, and in addition to inequality (5) let us also assume there is a number $C_{3}^{*}<\infty$ such that

$$
\|F(U, t)\| \leq C_{3}^{*}\left(\left\|A^{\nu_{1}^{*}} U\right\|+1\right)\left(\left\|A^{\nu_{2}^{*}} U\right\|+1\right)
$$

with $\nu_{1}^{*}+\nu_{2}^{*} \leq 1$ for all $U \in \widetilde{H}$.

We will first prove a collection of lemmas, which will then allow us to prove more abstract versions of Theorems 1 and 2 .

Lemma 3. Assume $U$ is a solution of $(E Q)$ on a time interval having $t$ as an interior point. Then

$$
\begin{gathered}
\frac{d}{d t}\left(\frac{1}{2}\|U(t)\|^{2}\right)+(1-\eta)\left\|A^{1 / 2} U(t)\right\|^{2} \leq C_{1}(t)\|U(t)\|^{2}+C_{2}(t), \\
\frac{d}{d t}\left(\frac{1}{2}\|U(t)\|^{2}\right) \leq\left(C_{1}(t)-\lambda_{0}(1-\eta)\right)\|U(t)\|^{2}+C_{2}(t)
\end{gathered}
$$

Proof. We multiply the equation

$$
U_{t}+A U=F(U, t)
$$


by $U$ and use $(A U, U)=\left\|A^{1 / 2} U\right\|^{2}$ together with inequality (4) to obtain

$$
\begin{aligned}
\frac{d}{d t}\left(\frac{1}{2}\|U(t)\|^{2}\right)+\| & A^{1 / 2} U(t) \|^{2} \\
& =(F(U, t), U)=(F(U, t), U)^{+}-(F(U, t), U)^{-} \\
& \leq(\eta-1)(F(U, t), U)^{-}+\eta\left\|A^{1 / 2} U(t)\right\|^{2}+C_{1}(t)\|U\|^{2}+C_{2}(t) .
\end{aligned}
$$

Thus,

$$
\frac{d}{d t}\left(\frac{1}{2}\|U(t)\|^{2}\right)+(1-\eta)\left\|A^{1 / 2} U(t)\right\|^{2} \leq C_{1}(t)\|U(t)\|^{2}+C_{2}(t),
$$

and, as $\left\|A^{1 / 2} U(t)\right\|^{2} \geq \lambda_{0}\|U(t)\|^{2}$,

$$
\frac{d}{d t}\left(\frac{1}{2}\|U(t)\|^{2}\right) \leq\left(C_{1}(t)-\lambda_{0}(1-\eta)\right)\|U(t)\|^{2}+C_{2}(t) .
$$

Lemma 4. Assume $U(t)$ is a solution of $(E Q)$ on the time interval $\left[t_{0}, t_{1}\right]$. Then

$$
\|U(t)\| \leq \exp \left(C_{6}\left(t-t_{0}\right)\right) \sqrt{\left\|U\left(t_{0}\right)\right\|^{2}+1}
$$

and

$$
(1-\eta) \int_{t_{0}}^{t}\left\|A^{1 / 2} U(\tau)\right\|^{2} d \tau \leq C_{6}\left(t-t_{0}\right)+\exp \left(2 C_{6}\left(t-t_{0}\right)\right)\left(\left\|U\left(t_{0}\right)\right\|^{2}+1\right)
$$

for $t \in\left[t_{0}, t_{1}\right]$.

Proof. From inequality (8) we get

which implies

$$
\frac{d}{d t}\left(\frac{1}{2}\|U(t)\|^{2}\right) \leq C_{6}\left(\|U(t)\|^{2}+1\right)
$$

and therefore

$$
\frac{d}{d t}\left(\|U(t)\|^{2}+1\right) \leq 2 C_{6}\left(\|U(t)\|^{2}+1\right)
$$

$$
\|U(t)\|^{2}+1 \leq \exp \left(2 C_{6}\left(t-t_{0}\right)\right)\left(\left\|U\left(t_{0}\right)\right\|^{2}+1\right)
$$

so

$$
\|U(t)\| \leq \exp \left(C_{6}\left(t-t_{0}\right)\right) \sqrt{\left\|U\left(t_{0}\right)\right\|^{2}+1} .
$$

This proves inequality (10). From inequality (8) we also have

and therefore

$$
\frac{d}{d t}\left(\frac{1}{2}\|U(t)\|^{2}\right)+(1-\eta)\left\|A^{1 / 2} U(t)\right\|^{2} \leq C_{6}\left(\|U(t)\|^{2}+1\right)
$$

$$
\|U(t)\|^{2}+2(1-\eta) \int_{t_{0}}^{t}\left\|A^{1 / 2} U(\tau)\right\|^{2} d \tau \leq \int_{t_{0}}^{t} 2 C_{6}\left(\|U(\tau)\|^{2}+1\right) d \tau+\left\|U\left(t_{0}\right)\right\|^{2} .
$$

Using inequality (10) we get

$$
2(1-\eta) \int_{t_{0}}^{t}\left\|A^{1 / 2} U(\tau)\right\|^{2} d \tau \leq 2 C_{6}\left(t-t_{0}\right)+\exp \left(2 C_{6}\left(t-t_{0}\right)\right)\left(\left\|U\left(t_{0}\right)\right\|^{2}+1\right),
$$

which immediately implies inequality (11). 
Now we turn to some lemmas which are more exclusively used to prove the abstract version of Theorem 2 .

LEMMA 5. Given $\varepsilon>0$ there is a $\delta>0$ such that for any $K_{1}, K_{2}<\infty$ there is a $\tau_{0}<\infty$ with the property that if $U$ solves $(E Q)$ on an interval $[0, T],\|U(0)\| \leq K_{2}$, and $C_{1}(t), C_{2}(t) \leq \delta$ for $t \geq K_{1}$, then $\|U(t)\| \leq \varepsilon$ for all $t \in\left[K_{1}+\tau_{0}, T\right]$.

Proof. Choosing $\delta \leq \frac{1}{2}(1-\eta) \lambda_{0}$ we obtain for $t \geq K_{1}$ using inequality (9), that

$$
\frac{d}{d t}\left(\frac{1}{2}\|U(t)\|^{2}\right) \leq-\frac{1}{2}(1-\eta) \lambda_{0}\|U(t)\|^{2}+\delta .
$$

This means that $\|U(t)\|$ decreases unless $\|U(t)\| \leq \sqrt{\frac{2 \delta}{(1-\eta) \lambda_{0}}}$. Therefore

$$
\left\{t \in\left[K_{1}, \infty\right):\|U(t)\| \geq 2 \sqrt{\frac{2 \delta}{(1-\eta) \lambda_{0}}}\right\}
$$

is an interval, and on this interval

$$
\frac{d}{d t}\left(\frac{1}{2}\|U(t)\|^{2}\right) \leq-\frac{1}{4}(1-\eta) \lambda_{0}\|U(t)\|^{2} .
$$

Thus

$$
\|U(t)\| \leq \exp \left(-(1-\eta) \lambda_{0}\left(t-K_{1}\right) / 4\right)\left\|U\left(K_{1}\right)\right\|
$$

on this interval, and from the previous lemma $\left\|U\left(K_{1}\right)\right\|$ is of course bounded independent of the specific solution, and we obtain in any case

$$
\|U(t)\| \leq \max \left(\exp \left(-(1-\eta) \lambda_{0}\left(t-K_{1}\right) / 4\right)\left\|U\left(K_{1}\right)\right\|, 2 \sqrt{2 \delta\left((1-\eta) \lambda_{0}\right)^{-1}}\right) .
$$

So, if we choose $\delta$ small enough to make $2 \sqrt{2 \delta\left((1-\eta) \lambda_{0}\right)^{-1}} \leq \varepsilon$, then there will be a $\tau_{0}$ such that for all $t \geq \tau_{0}+K_{1}$ we have $\|U(t)\| \leq \varepsilon$.

LEMMA 6. Given $\varepsilon>0$ there is a $\delta>0$ such that if $K_{1}, K_{2}<\infty$ and $C_{1}(t), C_{2}(t) \leq \delta$ for $t \geq K_{1}$ then with the $\tau_{0}$ from Lemma 5 for every solution $U$ of $(E Q)$ with $\|U(0)\| \leq$ $K_{2}$ on an interval $[0, T]$ containing at least $\left[0, K_{1}+\tau_{0}+\varepsilon\right]$, we have that for every $t_{0} \in\left[K_{1}+\tau_{0}, T-\varepsilon\right]$ there exists a $t \in\left[t_{0}, t_{0}+\varepsilon\right]$ with $\left\|A^{1 / 2} U(t)\right\| \leq \varepsilon$.

Proof. Integrating inequality (8) from $t_{0}$ to $t_{0}+\varepsilon$ we obtain

$$
\begin{aligned}
\int_{t_{0}}^{t_{0}+\varepsilon}\left\|A^{1 / 2} U(\tau)\right\|^{2} d \tau(1-\eta) & \leq \int_{t_{0}}^{t_{0}+\varepsilon}\left(C_{1}(\tau)\|U(\tau)\|^{2}+C_{2}(\tau)\right) d \tau+\left\|U\left(t_{0}\right)\right\|^{2} \\
& \leq \delta\left(\int_{t_{0}}^{t_{0}+\varepsilon}\|U(\tau)\|^{2} d \tau+1\right)+\left\|U\left(t_{0}\right)\right\|^{2} .
\end{aligned}
$$

As, by choosing $\delta$ arbitrarily small, we can make the latter quantity as small as we please, there has to be a $t \in\left[t_{0}, t_{0}+\varepsilon\right]$ such that $\left\|A^{1 / 2} U(t)\right\| \leq \varepsilon$.

LEMMA 7. For every $C<\infty$ there is an $\varepsilon>0$ such that if $U$ is a solution of $(E Q)$ on the interval $\left[t_{1}, t_{2}\right]$ with $t_{2} \leq t_{1}+\varepsilon$, then $\left\|A^{1 / 2} U\left(t_{1}\right)\right\| \leq C$ implies $\left\|A^{1 / 2} U(t)\right\| \leq 2 C$ for $t \in\left[t_{1}, t_{2}\right]$.

Pr o of. It is easy to see that the proof of Corollary 2.3 in [15] can be adapted to the situation prevailing here. 
Lemma 8. For any initial value $U_{0} \in D\left(A^{\mu}\right)$ there always is exactly one solution $U(t)$ of $(E Q)$ with $U\left(t_{0}\right)=U_{0}$ on an interval $\left[t_{0}, t_{1}\right]$ for some $t_{1}>t_{0}$, and if $\left[t_{0}, t_{1}\right)$ is its maximal interval of existence, and $t_{1}<\infty$, then the quantity $\left\|A^{1 / 2} U(t)\right\|$ cannot be bounded. Also if $U$ and $V$ are two solutions of $(E Q)$ on the interval $\left[t_{0}, t_{2}\right]\left(t_{0}<t_{2}<\infty\right)$ with

$$
\left\|A^{\mu} U(t)\right\|+\left\|A^{\mu} V(t)\right\| \leq M
$$

on this interval, then there is a constant $C$ only depending on $M$, such that

$$
\left\|A^{\mu}(U(t)-V(t))\right\| \leq \exp \left(C\left(t-t_{0}\right)\right)\left\|A^{\mu}\left(U\left(t_{0}\right)-V\left(t_{0}\right)\right)\right\|
$$

for $t \in\left[t_{2}, t_{0}\right]$. If $U_{0} \in \widetilde{H}$ then the solution $U(t)$ belongs to $\widetilde{H}$ also.

Pro of. Using Theorem 14.1 (p. 159) in [1] we can easily see that for any solution $U$ the function $F(U(t), t)$ is at least locally Hölder continuous in $\left(t_{0}, t_{1}\right)$. Then from Theorem 3.1 (p. 109) also in [1] we can see that $U$ also solves the variation-of-constants formula

$$
U(t)=\exp \left(-\left(t-t_{0}\right) A\right) U_{0}+\int_{t_{0}}^{t} \exp (-(t-s) A) F(U(s), s) d s .
$$

As is well known and can be seen using the theorems already quoted, every solution of this integral equation in $C^{0}\left(\left[t_{0}, t_{1}\right], D\left(A^{\mu}\right)\right)$ is also a solution of $(E Q)$. We can obtain such solutions as fixed points of the mapping $C_{U_{0}}: C^{0}\left(\left[t_{0}, t_{1}\right], D\left(A^{\mu}\right)\right) \rightarrow C^{0}\left(\left[t_{0}, t_{1}\right], D\left(A^{\mu}\right)\right)$ given by

$$
C_{U_{0}}(U)=\exp \left(-\left(t-t_{0}\right) A\right) U_{0}+\int_{t_{0}}^{t} \exp (-(t-s) A) F(U(s), s) d s .
$$

As we will see it is a local contraction in a neighborhood of the function $U(t) \equiv U_{0}$ in the Banach space $C^{0}\left(\left[t_{0}, t_{1}\right], D\left(A^{\mu}\right)\right)$ with the norm

$$
\max _{\tau \in\left[t_{0}, t_{1}\right]}\left[\left\|A^{\mu} U(\tau)\right\|\right]
$$

provided the time interval is chosen short enough. The Banach fixed point theorem then gives us existence and uniqueness. To see we have a contraction consider $U_{1}$ and $U_{2}$, and let $V_{i}=C_{U_{i}(0)}\left(U_{i}\right)$. Also let $W=V_{1}-V_{2}$, and $\widehat{W}=U_{1}-U_{2}$. Then by inequality (6),

$$
\left\|W_{t}(t)+A W(t)\right\| \leq C_{5}\left(\left\|A^{\mu} V_{1}(t)\right\|+\left\|A^{\mu} V_{2}(t)\right\|\right)\left\|A^{\mu} \widehat{W}(t)\right\| .
$$

As we also have

$$
W(t)=\exp (-t A) W(0)+\int_{t_{0}}^{t} \exp (-(t-s) A)\left(W_{t}+A W\right) d s,
$$

we get with $\widehat{M}=2 \max _{\left[t_{0}, t_{1}\right], i=1,2}\left(\left\|A^{\mu} V_{i}(t)\right\|\right)$ that

$$
\left\|A^{\mu} W(t)\right\| \leq C\left\|A^{\mu} W(0)\right\|+C C(\widehat{M}) \int_{t_{0}}^{t}(t-s)^{-\mu}\left\|A^{\mu} \widehat{W}(s)\right\| d s .
$$

This makes the fact that we have a contraction clear as for fixed initial values the first expression is zero. The local stability inequality (12) then also follows easily.

Now using an argument analogous to that of Lemma 2.2 in [15] it is easy to see that a solution can only cease to exist if $\left\|A^{1 / 2} U(t)\right\|$ goes to infinity. 
If $U(t)$ is a solution then of course $U_{t}+A U=F(U, t)$, and so $L_{\omega} U_{t}+L_{\omega} A U=$ $L_{\omega} F(U, t)$ and $\left(L_{\omega} U\right)_{t}+A L_{\omega} U=F\left(L_{\omega} U, t\right)$ by our assumptions. So $L_{\omega} U(t)$ is also a solution, and if $L_{\omega} U(0)=U(0)$, which is true if $U(0) \in \widetilde{H}$, then by uniqueness also $L_{\omega} U(t)=U(t)$.

The following lemma is familiar from the theory of ordinary differential equations.

Lemma 9. Assume a function $U \in C^{0}((0, T], D(A)) \cap C^{0}\left([0, T], D\left(A^{\mu}\right)\right)$ solves $(E Q)$. Then there is an $\varepsilon>0$ such that the solution $V(t)$ with initial value $V(0)=V_{0}$ exists on $[0, T]$ also if $\left\|A^{\mu}\left(V_{0}-U(0)\right)\right\|<\varepsilon$.

Proof. Let $M=2 \max _{t \in[0, T]}\left\|A^{\mu} U(t)\right\|$. For an arbitrary $\varepsilon<M / 2$ we can find at least a $T_{C}>0$ such that for $t \in\left[0, T_{C}\right]$ the solution $V$ exists and fulfills $\left\|A^{\mu} V(t)\right\| \leq M$. Then from (12) we get, as the time interval we consider is finite,

$$
\left\|A^{\mu}(U(t)-V(t))\right\| \leq C\left\|A^{\mu}(U(0)-V(0))\right\|
$$

at least as long as $\left\|A^{\mu} V(t)\right\| \leq M$. Taking $\varepsilon \leq \frac{M}{2 C}$ we see that $\left\|A^{\mu} V(t)\right\| \leq \frac{3}{4} M$ if $\left\|A^{\mu} V(t)\right\| \leq M$. For continuity reasons this is then true as long as $V$ exists. So $V$ can never cease to exist on this interval.

The following is an abstract version of Theorem 1 . We prove it by the same method as in [2].

Theorem 10. Assume $U_{0} \in \widetilde{H}$. Then there is exactly one solution of $(E Q)$ and it exists for all times.

Proof. By Lemma 8 the solution $U(t)$ stays in $\widetilde{H}$ as long as it exists. Therefore inequality (7) is valid for all $t$. It stays valid of course if we enlarge $\nu_{1}$ or $\nu_{2}$, though perhaps with a different constant, so we can assume $\nu_{1}+\nu_{2}=1$. The case $\nu_{1}=\nu_{2}=1 / 2$ is left to the reader. So assume now $\nu_{1}<1 / 2<\nu_{2}$. Then, as

$$
\begin{aligned}
& \left\|A^{\nu_{1}} U\right\| \leq C\|U\|^{1-2 \nu_{1}}\left\|A^{1 / 2} U\right\|^{2 \nu_{1}}, \\
& \left\|A^{\nu_{2}} U\right\| \leq C\left\|A^{1 / 2} U\right\|^{2\left(1-\nu_{2}\right)}\|A U\|^{2 \nu_{2}-1},
\end{aligned}
$$

we obtain

$$
\|F(U, t)\| \leq C\left(\|U\|^{1-2 \nu_{1}}+1\right)\left(\left\|A^{1 / 2} U\right\|^{2+2\left(\nu_{1}-\nu_{2}\right)}+1\right)\left(\|A U\|^{2 \nu_{2}-1}+1\right),
$$

and

$$
|(F(U, t), A U)| \leq C\left(\|U\|^{1-2 \nu_{1}}+1\right)\left(\left\|A^{1 / 2} U\right\|^{2+2\left(\nu_{1}-\nu_{2}\right)}+1\right)\left(\|A U\|^{2 \nu_{2}}+1\right) .
$$

Using Young's inequality we get

$$
\begin{aligned}
& \left(\|U\|^{1-2 \nu_{1}}+1\right)\left(\left\|A^{1 / 2} U\right\|^{2+2\left(\nu_{1}-\nu_{2}\right)}+1\right)\left(\|A U\|^{2 \nu_{2}}+1\right) \\
& \quad \leq \varepsilon\|A U\|^{2}+C(\varepsilon)\left(\|U\|^{\frac{1-2 \nu_{1}}{\nu_{1}}}+1\right)\left(\left\|A^{1 / 2} U\right\|^{\frac{2+2\left(\nu_{1}-\nu_{2}\right)}{\nu_{1}}}+1\right) .
\end{aligned}
$$

Now $\frac{2+2\left(\nu_{1}-\nu_{2}\right)}{\nu_{1}}=\frac{2+2 \nu_{1}-2\left(1-\nu_{1}\right)}{\nu_{1}}=4$, and so we get, using inequality (10),

$$
\begin{aligned}
|(F(U, t), A U)| & \leq \varepsilon\|A U\|^{2}+C(\varepsilon)\left(\|U\|^{\frac{1-2 \nu_{1}}{\nu_{1}}}+1\right)\left(\left\|A^{1 / 2} U\right\|^{4}+1\right) \\
& \leq \varepsilon\|A U\|^{2}+C(\varepsilon, U(0))\left(\left\|A^{1 / 2} U\right\|^{4}+1\right) .
\end{aligned}
$$


Multiplying $(E Q)$ by $A U(t)$ we obtain then

$$
\frac{d}{d t}\left(\frac{1}{2}\left\|A^{1 / 2} U(t)\right\|^{2}\right)+\|A U(t)\|^{2}=(F(U, t), A U) \leq \frac{1}{2}\|A U\|^{2}+C\left(\left\|A^{1 / 2} U\right\|^{4}+1\right) .
$$

Thus

$$
\frac{d}{d t}\left(\left\|A^{1 / 2} U(t)\right\|^{2}+1\right) \leq C\left(\left\|A^{1 / 2} U\right\|^{4}+1\right) \leq C\left(\left\|A^{1 / 2} U\right\|^{2}+1\right)^{2}
$$

We divide by $\left\|A^{1 / 2} U\right\|^{2}+1$ and obtain

So

$$
\frac{d}{d t} \log \left(\left\|A^{1 / 2} U(t)\right\|^{2}+1\right) \leq C\left(\left\|A^{1 / 2} U(t)\right\|^{2}+1\right) .
$$

$$
\log \left(\left\|A^{1 / 2} U(t)\right\|^{2}+1\right) \leq C \int_{0}^{t}\left(\left\|A^{1 / 2} U(s)\right\|^{2}+1\right) d s+\log \left(\left\|A^{1 / 2} U(0)\right\|^{2}+1\right) .
$$

From inequality (11) we also know that on any finite interval $[0, T]$ we have

$$
\int_{0}^{t}\left\|A^{1 / 2} U(s)\right\|^{2} d s \leq C
$$

for all $t \in[0, T]$ with a fixed constant $C<\infty$. This gives us the estimate

$$
\left\|A^{1 / 2} U(t)\right\|^{2} \leq C
$$

for $t \in[0, T]$, and with Lemma 8 we obtain our claim.

Theorem 11. There is a number $\delta>0$ such that if $\max \left(C_{1}(t), C_{2}(t)\right) \leq \delta$ for $t \geq K_{1}$ with some $K_{1}<\infty$, then every solution $U(t)$ of $(E Q)$ on $[0, \infty)$ has the property that there is an $\varepsilon>0$ such that if $W_{0} \in D(A)$ and $\left\|A\left(W_{0}-U(0)\right)\right\|<\varepsilon$, then the solution $W(t)$ of $(E Q)$ with initial value $W_{0}$ also exists for all times.

Pro of. Combining Lemma 6 and Lemma 7 we can see that if $\delta$ is chosen sufficiently small, then any solution which exists at least until the time $K_{1}+\tau_{0}+1$ cannot cease to exist anymore. By Lemma 9 we can see that we can assure existence up to this point by starting out sufficiently close to $U(0)$.

3. Proof of Theorems 1 and 2. In order to prove Theorems 1 and 2 we now only have to relate their hypotheses to those of Sec. 2 and then apply Theorems 10 and 11. For this we will initially only use the Sobolev imbedding theorem in 3-space (see Theorem 4.6.2 in [17]).

The role of $A$ is largely the same in the introduction and Sec. 2, and the nonlinearity $F:[0, \infty) \times H_{5}^{1}(V) \rightarrow H$ can easily be restricted to $D\left(A^{9 / 10}\right)$, as $D\left(A^{9 / 10}\right) \subset H_{2}^{9 / 5}(V) \subset$ $H_{5}^{1}(V)$ and $\|U\|_{H_{5}^{1}} \leq C\left\|A^{9 / 10} U\right\|$. (See Theorem 1.15.3, (p.103) and Theorem 4.3.2.2 (p. 318 ) in [17].) Now inequality (1) is the same as inequality (4), and from the theorems just quoted we can immediately get (6) with $\mu=9 / 10$ from (3). In the same way we can prove (5) from (2) as with $\nu_{1}=3 / 8$ and $\nu_{2}=7 / 8$ we have $\nu_{1}+\nu_{2}=10 / 8<3 / 2$, as also

$$
\|U\|_{L_{4}} \leq C\left\|A^{3 / 8} U\right\|, \quad\|U\|_{H_{4}^{1}} \leq C\left\|A^{7 / 8} U\right\| .
$$

Now we also need to prove (7) for elements of $\widetilde{H}$. This is made possible by 
LEMMA 12. There is a constant $C$ such that

$$
\|U\|_{L_{4}} \leq C\|U\|_{H_{2}^{1 / 2}} \quad \text { and } \quad\|U\|_{H_{4}^{1}} \leq C\|U\|_{H_{2}^{3 / 2}}
$$

for all $U \in H_{2}^{3 / 2}(M)$ with $L_{\omega} U=U(\omega \in \mathbb{R})$.

Proof. Let $m_{0} \in M$ be an arbitrary point. We can introduce a chart $\mathcal{T}: O \rightarrow M$, where $O$ is either $B^{3}=\left\{x \in \mathbb{R}^{3}:|x|<1\right\}$ or $B \cap \mathbb{R}^{3+}$, for a neighborhood of $m_{0}$ so that $\mathcal{T}(0)=m_{0}$. As $\left(\widetilde{T}_{\omega}^{-1}\right)^{\prime}\left(m_{0}\right) \neq 0$, we can introduce a new coordinate system around $m_{0}$ by first choosing two vectors $v_{1}$ and $v_{2}$ which form a linearly independent system with the vector $\left.\frac{d}{d \omega}\right|_{\omega=0}\left(\mathcal{T}^{-1} \widetilde{T}_{\omega}^{-1} \mathcal{T}(0)\right)$, and then letting $X\left(y_{1}, y_{2}, y_{3}\right)=\widetilde{T}_{y_{1}}^{-1}\left(\mathcal{T}\left(y_{2} v_{1}+y_{3} v_{2}\right)\right)$ for $\left(y_{1}, y_{2}, y_{3}\right) \in \widetilde{O}$ for a suitable domain $\widetilde{O}$. Now for any function $U \in \widetilde{H}$ we have by definition of $\widetilde{H}$,

$$
T_{y_{1}} U\left(X\left(y_{1}, y_{2}, y_{3}\right)\right)=U\left(X\left(0, y_{2}, y_{3}\right)\right)
$$

Locally the expression $T_{y_{1}} U\left(X\left(y_{1}, y_{2}, y_{3}\right)\right)$ can of course be considered as a vector function, and therefore from the Sobolev imbedding theorem (Theorem 4.6.2 in [17]) for function spaces in $\mathbb{R}^{2}$ we get

$$
\left\|T_{y_{1}} U\left(X\left(y_{1}, y_{2}, y_{3}\right)\right)\right\|_{L_{4}(\widetilde{O})} \leq C\left\|T_{y_{1}} U\left(X\left(y_{1}, y_{2}, y_{3}\right)\right)\right\|_{H^{1 / 2}(\widetilde{O})} .
$$

As we have

$$
\left\|U\left(X\left(y_{1}, y_{2}, y_{3}\right)\right)\right\|_{H^{1}(\widetilde{O})} \leq C\left\|T_{y_{1}} U\left(X\left(y_{1}, y_{2}, y_{3}\right)\right)\right\|_{H^{1}(\widetilde{O})}
$$

and

$$
\left\|U\left(X\left(y_{1}, y_{2}, y_{3}\right)\right)\right\|_{L^{2}(\widetilde{O})} \leq C\left\|T_{y_{1}} U\left(X\left(y_{1}, y_{2}, y_{3}\right)\right)\right\|_{L^{2}(\widetilde{O})},
$$

by complex interpolation we immediately obtain

$$
\left\|U\left(X\left(y_{1}, y_{2}, y_{3}\right)\right)\right\|_{L_{4}(\widetilde{O})} \leq C\left\|U\left(X\left(y_{1}, y_{2}, y_{3}\right)\right)\right\|_{H^{1 / 2}(\widetilde{O})} .
$$

Then of course

$$
\|U\|_{L_{4}(X(\widetilde{O}))} \leq C\|U\|_{H^{1 / 2}(X(\widetilde{O}))},
$$

and it is easy to conclude the remainder by patching the neighborhoods together.

Now combining Theorem 1.15.3, (p. 103) and Theorem 4.3.2.2 (p. 318) of [17], we get inequality (7) of Lemma 12. This finally proves Theorem 1 and 2.

4. Some applications. In this section we discuss a class of applications in very general terms, and then choose a specific example of a system from magneto-hydrodynamics. The Navier-Stokes equation can be obtained from this by setting the initial magnetic field equal to zero. All the applications we have in mind are characterized by the fact that the vector bundle we consider is in fact trivial, and that the fibers consist of a collection of 3 -vectors and scalars, and that the group of transformations $T_{\omega}$ covers $\widetilde{T}_{\omega}(x)=O_{\omega} x+b_{\omega}$, where the $O_{\omega}$ are orthogonal matrices representing rotations, and the $b_{\omega}$ are vectors, and that $\mathcal{M}$ is actually a subset of $R^{3}$ with perhaps some boundaries glued together, to describe periodic flows in an infinite domain. The typical element of the bundle is then of the form $\left(x, s_{1}, . ., s_{m}, v_{1}, \ldots, v_{n}\right)^{T}$ where $x, v_{1}, \ldots, v_{n} \in \mathbb{R}^{3}$ and $s_{1}, \ldots, s_{m} \in \mathbb{R}$. Also the 
action of $T$ is described by

$$
T_{\omega}\left[\begin{array}{c}
x \\
s_{1} \\
: \\
s_{m} \\
v_{1} \\
: \\
v_{n}
\end{array}\right]=\left[\begin{array}{c}
O_{\omega} x+b_{\omega} \\
s_{1} \\
: \\
s_{m} \\
O_{\omega} v_{1} \\
: \\
O_{\omega} v_{n}
\end{array}\right] .
$$

This is of course the usual transformation rule for vectors and scalars under coordinate changes. If $U(x)=\left(x, s_{1}(x), \ldots, s_{m}(x), \ldots, v_{1}(x), \ldots, v_{n}(x)\right)^{T}$ is a section, then all elements of $\widetilde{H}$ must have the invariance property

$$
U(x)=\left[\begin{array}{c}
s_{1}\left(\widetilde{T}_{\omega}^{-1}(x)\right) \\
\vdots \\
s_{m}\left(\widetilde{T}_{\omega}^{-1}(x)\right) \\
O_{\omega} v_{1}\left(\widetilde{T}_{\omega}^{-1}(x)\right) \\
\vdots \\
O_{\omega} v_{n}\left(\tilde{T}_{\omega}^{-1}(x)\right)
\end{array}\right],
$$

which is what is usually expected to be true in any physical system with a rotationaltranslational symmetry. The verification of the equivariance of $A$ and $F$ with respect to $L_{\omega}$ is not usually difficult, and requires the same kind of calculations as one would make to show that these equations are invariant under translations and rotations of the coordinate system. Also self-adjoint principal parts are very common in the equations of mathematical physics, not just in quantum mechanics. Examples are the Laplace operator with Dirichlet boundary conditions and the Stokes operator.

Now we consider the equations of incompressible magneto-hydrodynamics with the boundary conditions of superconductivity. So let us assume $\Omega$ is a bounded subset of $\mathbb{R}^{3}$ with a smooth boundary. Then the equation are (see [7] and [8])

$$
\begin{aligned}
& v_{t}-\mu_{1} \Delta v+\nabla p=-v \cdot \nabla v-\frac{1}{8 \pi} \nabla \mathcal{H}^{2}+\frac{1}{4 \pi}(\mathcal{H} \cdot \nabla) \mathcal{H}+f \equiv F_{1}, \\
& \operatorname{div}(v)=0, \\
& \mathcal{H}_{t}-\mu_{2} \Delta \mathcal{H}=(v \cdot \nabla) \mathcal{H}+(\mathcal{H} \cdot \nabla) v \equiv F_{2}, \\
& \operatorname{div}(\mathcal{H})=0,
\end{aligned}
$$

in $\Omega$ with the boundary conditions

$$
v=0 \quad \text { and } \quad \mathcal{H} \cdot n=0, \quad \operatorname{curl}(\mathcal{H}) \times n=0
$$

on $\partial \Omega=S$ with the initial values

$$
v(x, 0)=v_{0}(x), \quad \mathcal{H}(x, 0)=\mathcal{H}_{0}(x) \quad(x \in \Omega) .
$$

Here $v(x, t)$ is the velocity field, $p(x, t)$ is the pressure, and $\mathcal{H}(x, t)$ is the magnetic field. Finally $\mu_{1}$ is the constant viscosity, and $\mu_{2}$ has the role of a magnetic viscosity. We must assume that $\operatorname{div}\left(v_{0}\right)=0$, and $\operatorname{div}\left(\mathcal{H}_{0}\right)=0$. To construct the Hilbert space $H$ we use here 
we need some building blocks. So let

$$
\mathrm{H}=\left\{u \in L_{2}(\Omega): \int_{\Omega} u \cdot \nabla \Psi d x=0 \text { for all } \Psi \in H_{2}^{1}(\Omega)\right\} .
$$

We assume that $\Omega$ is invariant under the group of transformations we are considering here. Let $P: L_{2}(\Omega) \rightarrow \mathrm{H}$ be the orthogonal projector. It is easy to see that $P$ commutes with all the transformations in question, as the space of gradients of functions on $\Omega$ is invariant under them. Then let $S=-P \Delta$ be the Stokes operator and $D(S)=\mathrm{H} \cap H_{20}^{1} \cap H_{2}^{2}$. It is elementary to check that $L_{\omega}(D(S))=D(S)$ and $L_{\omega} S=S L_{\omega}$. Also $S$ is well known to be self-adjoint (see, e.g., p. 104 in [16]). For the principal part of the equation for the magnetic field let $\Delta_{s}$ be the Laplace operator with

$$
D\left(\Delta_{s}\right)=\left\{\mathcal{H} \in \mathrm{H} \cap H_{2}^{2}: \operatorname{curl}(\mathcal{H}) \times n=0, \Delta \mathcal{H} \in \mathrm{H}\right\}
$$

as domain of definition. From Chap. III, Sec. 3.4 in [16] we can also see that this operator is self-adjoint. It of course has the same equivariance properties as $S$, as the normal vector $n(x)$ also does. Now let

$$
H=\mathrm{H} \times\left\{\mathcal{H} \in \mathrm{H}: \mathcal{H} \perp \operatorname{ker}\left(\Delta_{s}\right)\right\} .
$$

Then we can define our self-adjoint and equivariant operator as

$$
A=\left[\begin{array}{cc}
S & 0 \\
0 & -\Delta_{s}
\end{array}\right]
$$

with domain $D(A)=D(S) \times\left\{u \in D\left(\Delta_{s}\right): u \perp \operatorname{ker}\left(\Delta_{s}\right)\right\}$. Due to projecting out the kernel we get a positive operator. Now let $\mathcal{H}_{1}$ be the component of $\mathcal{H}_{0}$ in the kernel of $-\Delta_{s}$. One can easily see that this kernel only consists of functions $\mathcal{H}$ with $\operatorname{curl}(\mathcal{H})=0$. We can rewrite $\mathcal{H}$ as $\mathcal{H}(x, t)=\mathcal{H}_{1}(x)+\mathbf{H}(x, t)$. It is easy to see using the form $\operatorname{curl}(u \times \mathcal{H})$ of the nonlinearity in the third equation in (13) that $\mathcal{H}_{1}$ is also the projection of $\mathcal{H}(x, t)$ onto the kernel of $-\Delta_{s}$ as $F_{2}(v, \mathcal{H}) \perp \operatorname{ker}\left(-\Delta_{s}\right)$. So we can introduce $\mathbf{H}$ as the new variable for the magnetic field and define

$$
F(U, t)=\left[\begin{array}{c}
P F_{1}\left(v, \mathbf{H}+\mathcal{H}_{1}, t\right) \\
F_{2}\left(v, \mathbf{H}+\mathcal{H}_{1}, t\right)
\end{array}\right] \quad \text { for } U=\left[\begin{array}{c}
v \\
\mathbf{H}
\end{array}\right] .
$$

It is now a very elementary calculation to check that the equations have the claimed equivariance under all rotations and translations in general, and therefore under all helical motions, provided that the function $f$ and the initial value for $\mathcal{H}$ does. Inequality (1) follows in a way analogous to how the law of the conservation of energy is obtained. The additional properties are easily obtained by the fact that

$$
\|F(U, t)\|_{L_{2}} \leq C\left(\|U\|_{L_{4}}\|\nabla U\|_{L_{4}}+1\right) .
$$

Now assuming the required symmetry of the initial value, we get all the conclusions of Theorems 1 and 2. It should be pointed out that the equations of magneto-hydrodynamics with the boundary conditions used in [14] can be treated in a similar way.

Of course an example for a domain with one of the symmetries envisioned here is the torus

$$
\left\{(x, y, z):\left(\sqrt{x^{2}+y^{2}}-R_{1}\right)^{2}+z^{2}<R_{2}\right\} \quad\left(R_{1}>R_{2}\right)
$$


which is symmetric under all rotations around the $z$-axis. In contrast to [5] there is no need to confine the velocity vectors at any point to the plane through the point and the $x$-axis, as is done there. One can also consider the manifold obtained by identifying all points $(x, y, 0)$ with $(x, y, l)$ in the domain

$$
\left\{(x, y, z): \sqrt{x^{2}+y^{2}}<R, 0<z<l\right\},
$$

which describes a flow in an infinite pipe, which has both rotational and translational symmetries, which can be combined as helical symmetries. This is the situation considered in [10], and the existence part is covered here also for the Navier-Stokes and magnetohydrodynamics equations, but of course we do not study attractors here.

Finally $U$ does not necessarily have to represent the physical quantities themselves, but could rather be the difference to an energy-stable equilibrium with the right symmetry. Then it is easy to verify our assumptions, and thus to obtain the stability of all time-dependent symmetric solutions.

\section{References}

[1] A. Friedman, Partial Differential Equations, Krieger, Malabar, Fla., 1983.

[2] J. M. Ghidaglia, Etude d'écoulements de fluides visqueux incompressibles: comportement pour les grands temps et applications aux attracteurs, Thèse de 3e cycle, Université de Paris Sud, Orsay, 1984.

[3] M. W. Hirsch, Differential Topology, Springer, Berlin, 1976

[4] O. A. Ladyzhenskaya, Solution in the large of the nonstationary boundary value problem for the Navier-Stokes system with two space variables, Comm. Pure Appl. Math. 12 (1959) 427-433.

[5] _- On unique global solvability of three dimensional Cauchy problem for Navier-Stokes equations with rotational symmetry, Zap. Nauchn. Sem. LOMI 7 (1968) 155-177.

[6] -, The Mathematical Theory of Viscous Incompressible Flows, 2nd ed., Gordon and Breach, New York, 1969.

[7] L. Landau and E. Lifschitz, Mechanics of Continuous Media, Nauka, Moscow, 1954 (in Russian); English transl.: Pergamon Press, Oxford, 1959; new edition: Hydrodynamics, Nauka, Moscow, 1986 (in Russian), English transl.: Fluid Mechanics, Pergamon Press, Oxford, 1987.

[8] - - - Electrodynamics of Continuous Media, Nauka, Moscow, 1957 (in Russian).

[9] J. Leray, Sur le mouvement d'un liquide visqueux emplissant l'espace, Acta Math. 63 (1934), 193-248.

[10] A. Mahalov, E. S. Titi and S. Leibovich, Invariant helical subspaces for the NavierStokes equations, Arch. Rational Mech. Anal. 112 (1990), 193-222.

[11] G. Ponce, R. Racke, T. S. Sideris and E. S. Titi, Global stability of large solutions to the $3 d$ Navier-Stokes equations, preprint.

[12] V. A. Solonnikov, Estimates of the solutions of a non-stationary linearized system of Navier-Stokes equations, Trudy Mat. Inst. Steklov. 70 (1964), 213-317 (in Russian).

[13] - On boundary problems for linear parabolic systems of differential equations of general type, Trudy Mat. Inst. Steklov. 83 (1965) (in Russian); English transl.: Proc. Steklov Inst. Math. 83 (1967). 
[14] G. Ströhmer, About an initial-boundary value problem from magneto-hydrodynamics, Math. Z. 209 (1992), 345-362.

[15] —, An existence result for partially regular weak solutions of certain abstract evolution equations, with an application to magneto-hydrodynamics, ibid. 213 (1993), 373-385.

[16] R. Temam, Infinite-Dimensional Dynamical Systems in Mechanics and Physics, Springer, New York, 1988.

[17] H. Triebel, Interpolation Theory, Function Spaces, Differential Operators, NorthHolland, Amsterdam, 1978.

[18] W. von Wahl, The Equations of Navier-Stokes and Abstract Parabolic Equations, Vieweg, Braunschweig, 1985. 